\title{
ON MEAN-PERIODICITY
}

\author{
BY EDWIN J. AKUTOWICZ
}

Communicated by Henry Helson, February 22, 1968

Introduction. Wiener's classical results on the closure of the translates of a function belonging to a Lebesgue class lead to the notion of mean-periodicity: A function $f$ belonging to a given space $M$ of functions defined on a Euclidean space is said to be mean-periodic in the space $M$ if the closed linear span of the translates of $f$ falls short of the entire space $M$. This notion clearly depends very strongly upon the function space $M$ under consideration. The space $M$ most thoroughly studied to date is the space of all continuous functions on the real line $R$ under the topology of uniform convergence on compact sets [4], [5], [8].

It is the purpose of the present note to outline some new results concerning spectral analysis and synthesis in translation invariant subspaces connected with a certain space of functions holomorphic on $R$.

A certain function space and its dual. We consider at first the following space $M$. Let $f(x+i y)$ denote a function holomorphic in a closed strip $|y| \leqq 1 / h$ for some $h<\infty$ such that

$$
\sup _{|y| \leqq 1 / h}\left\|e^{|x| / k} f(x+i y)\right\|<\infty
$$

for every $k>0$, where the norm appearing is that of $L^{2}(R)$. For $h$ and $k$ fixed, let $S_{\mathbf{k}}^{h}$ denote the Banach space formed by all such functions $f$ with norm given by the expression (1). The space $M$ is then the set

$$
\bigcup_{h<\infty} \bigcap_{k>0} S_{k}^{h}
$$

with the natural topology

$$
M=\underset{h \rightarrow \infty}{\operatorname{ind} \lim } \underset{k \rightarrow 0^{+}}{\operatorname{proj}} \lim S_{k}^{h}
$$

The space $M$ resembles spaces considered by Gelfand and Silov [2], [3] and by Roumieu [7], but it does not coincide with any of these. The space $M$ does occur amongst the spaces investigated by Palamodov [6].

It turns out that $M$ possesses several convenient properties as a topological vector space. $M$ is a Montel space and hence reflexive. $M$ 
is bornological. $M$ is a regular inductive limit; that is, every bounded set in $M$ is contained in a space

$$
S^{n}=\underset{k \rightarrow 0^{+}}{\operatorname{proj}} \lim S_{k}^{n}
$$

for some $n>0$ and bounded in $S^{n}$. This latter fact allows us to make effective use of the strong topology in the dual space $M^{\prime}$, defined by uniform convergence on the bounded sets of $M$.

The Fourier transformation $\mathfrak{F}$ effects an isomorphic mapping of the space $M$ on

$$
\mathfrak{F} M=\underset{k \rightarrow \infty}{\operatorname{ind} \lim } \underset{h \rightarrow 0^{+}}{\operatorname{proj}} \lim S_{k}^{h}
$$

(Cf. [6, p. 328].)

The elements $\lambda$ belonging to the dual space $M^{\prime}$ have the following structure

$$
\lambda=\sum_{p, q} D^{p}\left[x^{q} g_{p q}(x)\right], \quad g_{p q} \in L^{2}(R),
$$

where the sum over nonnegative integers $p$ and $q$ is in general infinite and where

$$
H^{p} p ! K^{q} q !\left\|g_{p q}\right\|=O(1) \quad(p, q \rightarrow \infty)
$$

for all $H<\infty$ and some $K>0$. The series (2) converges strongly in $M^{\prime}$ and the action of $\lambda$ on a function of $M$ is according to the formula

$$
\langle\lambda, f\rangle=\sum_{p, q}(-1)^{p} \int_{R} x^{q} g_{p q}(x) D^{p} f(x) d x, \quad f \in M .
$$

The function

$$
z \rightarrow \phi_{\lambda}(z)=\sum_{p, q}(i z)^{p}\left(i D_{z}\right)^{q} \int_{R} \frac{G_{p q}(t)}{t-z} d t, \quad G_{p q}=\Im_{g q},
$$

(the indicator of Fantappie of $\mathfrak{F} \lambda$ ) is of importance because the Fourier transform ${ }^{1} \mathfrak{F} \lambda, \lambda \in M^{\prime}$, coincides with the linear form

$$
F \rightarrow \int \phi_{\lambda}(s) F(s) d s, \quad F=\mathfrak{F} f, \quad f \in M,
$$

where the integral is extended over two parallel lines in the upper and lower half-planes, situated outside of the strip $|y| \leqq 1 / K ; K=K_{\lambda}$ is

${ }^{1}$ Recall that the Fourier transform $F_{\lambda}$ is defined through $\left\langle F_{\lambda}, F_{f}\right\rangle=2 \pi\langle\lambda, f\rangle$, where $\mathcal{F}_{f}(s)=\int_{R} e^{i \theta x} f(x) d x,(f \in M)$. 
the constant associated with the distribution $\lambda$. We have here a means of handling effectively complex spectra contained in a horizontal strip about the real axis.

It is not permissible to identify $\mathscr{F} \lambda$ with $\phi_{\lambda}$ because several different functions $\phi_{\lambda}$ can define the same form (3) (cf. [7], [1]). However, any two such functions $\phi_{\lambda}$ must differ by an entire function of a certain class.

Mean-periodic distributions of $M^{\prime}$. We designate as invariant subspaces those closed subspaces of the dual space $M^{\prime}$ which are invariant under all translations by $\xi \in R$.

Proposition 1. If $V^{\prime} \neq(0)$ is an invariant subspace of $M^{\prime}$, then $V^{\prime}$ necessarily contains at least one character, $x \rightarrow e^{i s x}$, s complex.

The orthogonal space $\left(V^{\prime}\right)^{\perp}$ is an invariant subspace of $M$, and the spectrum of $V^{\prime}$ consists of all common zeros (with multiplicity) of the Fourier transforms $\Im f f$ when $f$ runs over $\left(V^{\prime}\right)^{\perp}$.

Proposition 2. The system of exponential-monomials contained in an invariant subspace $V^{\prime}$ has the property that no exponential-monomial of the system belongs to the closed linear span of the others.

This fact allows us to write down a formal series for each meanperiodic $\lambda \in M^{\prime}$ :

$$
\lambda \sim \sum_{k} \sum_{j=0}^{p_{k}-1} C_{j k}(i x)^{j} e^{i \varepsilon_{k} x}
$$

where $s_{k}$ denotes the points of the spectrum of $\lambda, p_{k}$ the multiplicity of $s_{k}$, and where the coefficients $C_{j k}$ are given through the following rule (L. Schwartz [8]):

$$
\begin{gathered}
C_{j k}=\left\langle\lambda, n_{j k}\right\rangle, \quad \Im n_{j k}=N_{j k}, \\
N_{j k}(s)=N(s)\left\{\text { polar part of }\left(s-s_{k}\right)^{j} / j ! N(s)\right\}, \quad j=0,1, \cdots, p_{k}-1, \\
N=\Im n, \quad\langle\lambda, n\rangle=0, \quad n \in M .
\end{gathered}
$$

A major result is that the $C_{j k}$ do not depend upon the choice of $n \in M$ orthogonal to $\lambda$, but are intrinsically connected with $\lambda$.

Proposition 3. The coefficients $C_{j k}$ are uniquely determined by the distribution $\lambda$ as follows:

$$
C_{j k}=\text { residue of } \phi_{\lambda}(s)\left(\left(s-s_{k}\right)^{i} / j !\right) \text { at } s=s_{k} \text {. }
$$

and $C_{j k}=0$ in all other cases. 
Proposition 4. Every mean-periodic distribution $\lambda$ belonging to the space $M^{\prime}$ is a strong limit of a generalised sequence of linear combinations of exponential monomials associated with the spectrum of $\lambda$.

The formal series (4) can be interpreted as an interpolation series and the remainder calculated. This calculation, taken with the above double characterisation of the coefficients $C_{j k}$, yields the more precise result:

THEOROM 5. $\lambda$ is a mean-periodic distribution belonging to $\mathrm{M}^{\prime}$, then the formal series (4) converges to $\lambda$ in the strong topology of $\mathrm{M}^{\prime}$.

Mean-periodic functions in the space $\mathfrak{F} M$. The cospectrum of a function belonging to the space $\mathfrak{F} M$ is the set of real zeros of its Fourier transform. The problem of spectral synthesis is to decide whether every function whose cospectrum contains the cospectrum of a given function $f$ is a limit of translations of $f$ in $\mathscr{F} M$. It is familiar that such questions reduce to questions of divisibility in the Fourier transform space, which is $M$ in the present case. Here it is possible to divide out the real zeros of a function of $M$ by a Blaschke product in a horizontal strip. The necessary minorations in order to have a quotient in $M$ result by considering the harmonic measure in a slit strip.

Proposition 6. Let $f$ belong to $\mathfrak{F} M$. Every function of $\mathfrak{F} M$ whose cospectrum contains the cospectrum of $f$ is a limit of a generalised sequence of linear combinations of translates of $f$.

\section{BIBLIOGRAPHY}

1. E. J. Akutowicz, Sur certaines distributions quasi-analytiques au sens de $S$. Bernstein, Ann. Sci. Ecole. Norm. Sup. 79 (1962), 71-91.

2. I. M. Gelfand et G. E. Silov, Quelques applications de la théorie des fonctions généralisées, J. Math. Pures Appl. 35(1956), 383-413.

3. - Verallgemeinerten Funktionen (Distributionen). II: Lineare topologische Räume, Räume von Grundfunktionen und verallgmeinerten Funktionen, Hochschulbücher für Mathematik, Bd. 48, VEB Deutscher Verlag, Berlin, 1962; English transl., Academic Press, New York (to appear).

4. J.-P. Kahane, Sur quelques problèmes d'unicite et de prolongement, relatifs aux fonctions approachables par des sommes d'exponentielles, Ann. Inst. Fourier, Grenoble 5 (1953-1954), 39-130.

5. - Lectures on mean-periodic functions, Tata Institute for Fundamental Research, Bombay, 1959.

6. V. P. Palamodov, Fourier transforms of infinitely differentiable functions of rapid growth, Trudy Moskov. Mat. Obšc. 11 (1962), 309-350. (Russian)

7. Ch. Roumieu, Sur quelques extensions de la notion de distribution, Ann. Sci. École Norm. Sup. 77 (1960), 41-121.

8. L. Schwartz, Théorie gênérale des fonctions moyenne-périodiques, Ann. Math. 48 (1947), 857-929.

Faculte des Sciences, Montpellier 